\title{
Sb-Doped Titanium Oxide: A Rationale for Its Photocatalytic Activity for Environmental Remediation
}

\author{
Massimo Zimbone, ${ }^{\dagger}$ Giuseppe Cacciato, ${ }^{\dagger}$ Luca Spitaleri, $^{\ddagger}$ Russell G. Egdell, ${ }^{, \S}$ Maria Grazia Grimaldi, ${ }^{*}, \|$ \\ and Antonino Gulino**\$。 \\ ${ }^{\dagger}$ CNR-IMM, via S. Sofia 64, 95123 Catania, Italy \\ ${ }^{\ddagger}$ Dipartimento di Scienze Chimiche and INSTM, UdR of Catania, Viale Andrea Doria 6, 95123 Catania, Italy \\ ${ }^{\S}$ Department of Chemistry, Inorganic Chemistry Laboratory, University of Oxford, South Parks Road, Oxford OX1 3QR, U.K. \\ "CNR-IMM, and Dipartimento di Fisica e Astronomia, Università di Catania, via S. Sofia 64, 95123 Catania, Italy
}

Supporting Information

\begin{abstract}
The problem of water purification is one of the most urgent issues in developing countries, where large infrastructures and energy resources are limited. Among the possibilities for a cheap route to clean water, photocatalytic materials in the form of coatings or nanostructures are among the most promising. The most widely studied photocatalytic material is titanium dioxide $\left(\mathrm{TiO}_{2}\right)$. Here, we investigate the photocatalytic properties of $1.5 \% \mathrm{Sb}$-doped $\mathrm{TiO}_{2}$ and laser-irradiated Sb-doped $\mathrm{TiO}_{x}$. Calcined Sb-doped $\mathrm{TiO}_{2}$ was found to adopt the rutile structure, but it turned amorphous after laser irradiation. Photocatalytic tests for $\mathrm{Sb}$-doped $\mathrm{TiO}_{2}$ showed an activity 1 order of magnitude higher than that of an undoped $\mathrm{TiO}_{2}$ control sample under both ultraviolet and visible irradiation. A further sizeable enhancement resulted from laser irradiation. The increased photocatalytic activity is ascribed to both

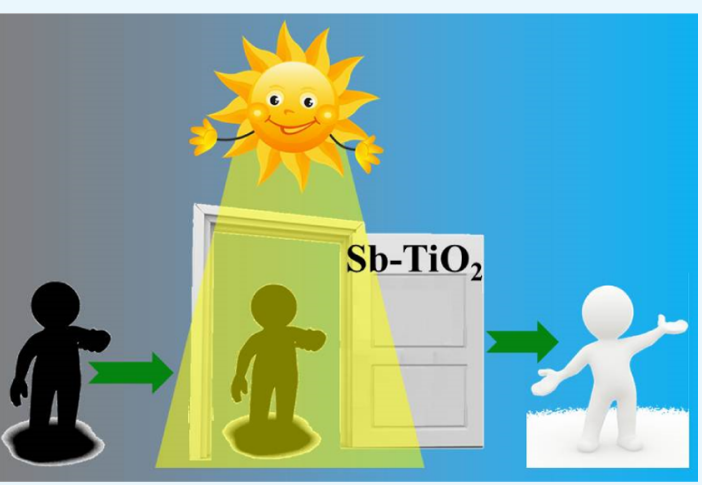
enhanced visible region absorption associated with $\mathrm{Sb}$-induced lone pair surface electronic states and trapping of the holes at the lone pair surface sites, thus inhibiting the recombination of the electrons and holes generated in the initial photoexcitation step. This study shows the first rationalization of the photocatalytic properties of $\mathrm{Sb}-\mathrm{TiO}_{2}$ in terms of its electronic structure.
\end{abstract}

\section{INTRODUCTION}

There is an increasing attention in the synthesis, characterization, and properties of wide-gap oxide semiconductors able to bring about the photocatalytic reactions using sunlight. Photocatalysis involves excitation of a valence electron into the conduction band: the excited electrons can reduce the surfaceadsorbed species, whereas the valence band (VB) holes act as oxidizing agents. The photocatalytic efficiency depends on the separation of the photogenerated electrons and holes, so as to minimize the simple recombination. Titanium dioxide $\left(\mathrm{TiO}_{2}\right)$ is one of the most important materials in this field. ${ }^{1-6} \mathrm{TiO}_{2}$ has two important polymorphs: anatase and rutile with band gaps at low temperatures of 3.46 and $3.06 \mathrm{eV}$, respectively. These gaps fall in the near-ultraviolet (UV) region and, as a consequence, neither oxide can bring about the photocatalytic reactions under visible light. ${ }^{13-15}$

Following the observation of Asahi et al. ${ }^{1}$ that nitrogen doping in anatase $\mathrm{TiO}_{2}$ promotes the visible region photocatalytic activity, ${ }^{2,3,16-22}$ a range of other anionic dopants have been investigated including $\mathrm{B}^{23,24} \mathrm{C},{ }^{25,26}$ and $\mathrm{S}^{27,28}$ These elements are all less electronegative than oxygen, and consequently, the dopant-induced np states appear above the main oxygen $\mathrm{O} 2 \mathrm{p}$ VB. Transition-metal dopants including the group 5 elements $\mathrm{V},{ }^{29} \mathrm{Nb},{ }^{30}$ and $\mathrm{Ta}^{31}$ as well as the first-row elements such as $\mathrm{Cr},{ }^{31} \mathrm{Fe},{ }^{31}$ and $\mathrm{Ni}^{30}$ have also been shown to promote a visible light response, associated with the introduction of occupied $d$ states into the bulk band gap. Catalysts involving group IX elements including $\mathrm{Rh}$ (III) codoped with group $\mathrm{XV}$ elements such as $\mathrm{Sb}(\mathrm{V})$ to maintain the charge balance are also of current interest. ${ }^{32}$

Finally, post-transition metals including $\mathrm{Ga},{ }^{33} \mathrm{Sn},{ }^{7,34}$ and $\mathrm{Sb}^{35}$ have been explored as dopants. A remarkable enhancement in the photocatalytic activity associated with $\mathrm{Sn}$ doping is somewhat surprising as $\mathrm{Sn}(\mathrm{IV})$ is an isovalent dopant in $\mathrm{TiO}_{2}$ and would not be expected to introduce states into the band gap. However, it has been shown that $\mathrm{Sn}$ segregates to the surface of $\mathrm{TiO}_{2}$ as $\mathrm{Sn}(\mathrm{II})$ and "lone pair" surface states associated with the segregated dopant sit above the main VB edge. Transitions from these states into the conduction band promote the visible region absorption, and at the same time, the resulting holes are trapped in the lone pair orbitals protruding from the surface.

Received: June 26, 2018

Accepted: September 5, 2018

Published: September 17, 2018 
$\mathrm{Sb}$ doping is more complicated. In $\mathrm{Sb}$-doped $\mathrm{SnO}_{2}$, bulk $\mathrm{Sb}(\mathrm{V})$ acts as a simple one-electron donor, and degenerate $\mathrm{n}$ type doping to give a transparent conducting oxide is easily achieved. ${ }^{36-38} \mathrm{Sb}$ doping in the anatase leads to a similar behavior, with a pronounced increase in the conductivity as electrons are introduced into the conduction band. ${ }^{39}$ In both the systems, segregation of $\mathrm{Sb}$ (III) to the surface sites-similar to $\mathrm{Sn}$ (II) segregation-is also observed. However, Sb doping in the rutile phase of $\mathrm{TiO}_{2}$ leads to the compensation of incorporation of $\mathrm{Sb}(\mathrm{V})$ into the bulk sites by the formation of cation vacancies, and so this phase remains nonconducting. ${ }^{40-42}$ Again, there is a segregation of $\mathrm{Sb}$ (III) to the surface sites, and lone pair surface states may be seen above the VB edge in photoemission experiments. ${ }^{40}$

Obviously, $\mathrm{TiO}_{2}$-based materials show many other important properties, and, for example, some of them are related to the nanomedicine (angiogenesis, anticancer, drug delivery, properties, etc.), but in the present study, we focused our attention to photocatalysis. ${ }^{43-45}$

In fact, the similarities between the Sn-doped and the Sbdoped rutile in the present paper lead us to explore the photocatalytic activity of the Sb-doped material. In order to help unravel the contributions of enhanced visible region absorption and hole trapping at the surface to the photocatalytic activity, dye degradation experiments involving both visible and UV irradiation were performed.

As an alternative to doping, there has been a recent interest in the "physical" manipulation of surfaces as a means toward the enhancement of catalytic activity, for example by reduction in hydrogen ${ }^{46,47}$ and by laser irradiation of surfaces. ${ }^{48-51}$ Here, we combine the two approaches by exploring the photocatalytic activity of laser-ablated $\mathrm{Sb}$-doped $\mathrm{TiO}_{2}$.

\section{RESULTS AND DISCUSSION}

Rutherford Backscattering Spectrometry. Figure 1 shows the Rutherford backscattering spectrometry (RBS)

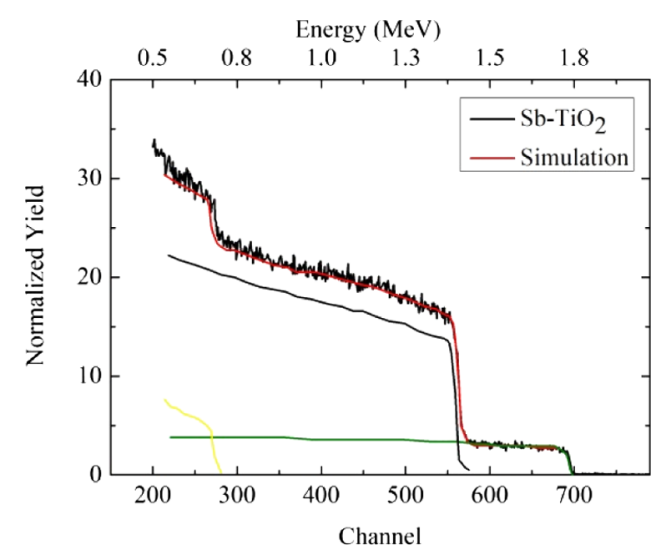

Figure 1. RBS spectrum (black line) of the $\mathrm{Sb}$-doped $\mathrm{TiO}_{2}$ sample. The steps at channels 700, 560, and 260 are related to the $\mathrm{Sb}, \mathrm{Ti}$, and $\mathrm{O}$ atoms, respectively. The horizontal profile between channels 590 and 700 indicates that the $\mathrm{Sb}$ concentration is uniform into the $\mathrm{TiO}_{2}$ matrix on the length scale of RBS. The red line shows the simulation of the overall RBS spectrum that confirms the $\sim 1.5$ atom $\%$ amount of $\mathrm{Sb}$ in the matrix and the $[\mathrm{Ti}] /[\mathrm{O}]=1: 2$ stoichiometry. Green, yellow, and blue lines represent the simulated signals for the $\mathrm{Sb}, \mathrm{Ti}$, and $\mathrm{O}$ species, respectively. Similar results have been obtained for the $\mathrm{Sb}$ doped $\mathrm{TiO}_{x}$ sample, thus demonstrating that the overall stoichiometry is unaffected by the laser irradiation. spectrum of the Sb-doped $\mathrm{TiO}_{2}$ sample. Taking into account the atomic numbers of $\mathrm{Sb}$ and $\mathrm{Ti}$, the RBS signal indicates that 1.3 atom $\%$ of $\mathrm{Sb}$ is incorporated into the $\mathrm{TiO}_{2}$ matrix, which is very close to the nominal $1.5 \%$ doping level. RBS also confirms the $[\mathrm{Ti}] /[\mathrm{O}]=1: 2$ overall stoichiometry. The simulated signals for $\mathrm{Sb}$ (green), $\mathrm{Ti}$ (yellow), and $\mathrm{O}$ (blue) atoms are also shown. Similar results have been obtained for the $\mathrm{Sb}$ doped $\mathrm{TiO}_{x}$ samples, demonstrating that the overall bulk stoichiometry is unaffected by the laser irradiation, although there are obviously changes at the surface.

$\mathrm{X}$-ray Diffraction. Figure 2 shows the X-ray diffraction (XRD) patterns for $\mathrm{Sb}-\mathrm{TiO}_{2}$ and $\mathrm{Sb}-\mathrm{TiO}_{x}$ samples. The

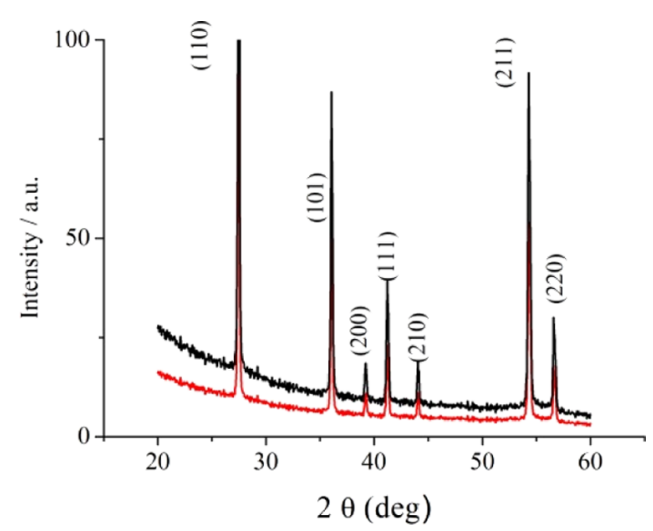

Figure 2. XRD diffraction patterns for $\mathrm{Sb}-\mathrm{TiO}_{2}$ (black line) and $\mathrm{Sb}-$ $\mathrm{TiO}_{x}$ (red line).

(110), (101), (200), (111), (210), and (211) reflections of rutile are evident. ${ }^{52}$ It is well-known that the rutile structure is thermodynamically favored for large crystallites and is almost always observed after annealing $\mathrm{TiO}_{2}$ samples at a temperature greater than about $600{ }^{\circ} \mathrm{C} .{ }^{53,54}$

Sharp diffraction peaks are recognized for both Sb-doped $\mathrm{TiO}_{2}$ and $\mathrm{Sb}$-doped $\mathrm{TiO}_{x}$. However, the decrease in the intensity of the X-ray reflections after laser irradiation to give Sb-doped $\mathrm{TiO}_{x}$ can be linked to the formation of an amorphous layer on the sample surface (vide infra).

The transmission electron microscopy (TEM) measurements of $\mathrm{Sb}$-doped $\mathrm{TiO}_{2}$ have already been performed, and the lattice image of crystals confirmed the XRD crystal structure. ${ }^{40}$

Raman Spectroscopy. The Raman spectra are shown in Figure 3. The $\mathrm{TiO}_{2}$ spectrum shows the main features of the rutile phase consisting in two peaks at 448 and $610 \mathrm{~cm}^{-1}$,

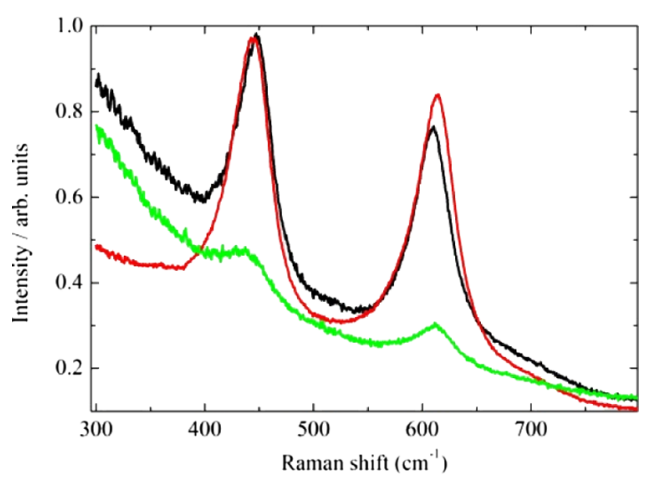

Figure 3. Raman spectra for $\mathrm{TiO}_{2}$ (black line), Sb-doped $\mathrm{TiO}_{2}$ (red line), and $\mathrm{Sb}$-doped $\mathrm{TiO}_{x}$ (green line). 
respectively. The former $\left(\mathrm{E}_{\mathrm{g}}\right.$ at $\left.448 \mathrm{~cm}^{-1}\right)$ is related to the doubly degenerate breathing vibration of the oxygen octahedron, whereas the latter $\left(A_{1 g}\right.$ at $\left.610 \mathrm{~cm}^{-1}\right)$ is related to the in-phase oxygen displacement.

The position of the peaks shifts slightly upon $\mathrm{Sb}$ doping or irradiating the samples. In particular, the $\mathrm{E}_{\mathrm{g}}$ peak shifts from 448 to $443 \mathrm{~cm}^{-1}$ with $\mathrm{Sb}$ incorporation and to $440 \mathrm{~cm}^{-1}$ after irradiation. The lower the frequency, the lower the force constant characteristic of the $\mathrm{Ti}-\mathrm{O}$ bonds. The presence of $\mathrm{Sb}$ may soften the structure by relaxing the bonds, thus creating intrinsic defects. ${ }^{55}$ In fact, even though the average $\mathrm{Ti}(\mathrm{IV})-\mathrm{O}$ bond length in $\mathrm{TiO}_{2}$ is $1.949 \AA$, shorter than the $\mathrm{Sb}(\mathrm{V})-\mathrm{O}$ bond length $(1.987 \AA)$ found in the mixed valence $\mathrm{Sb}_{2} \mathrm{O}_{4}$ compound that contains both $\mathrm{Sb}(\mathrm{V})$ and $\mathrm{Sb}(\mathrm{III})$ centers, the $\mathrm{Sb}-\mathrm{TiO}_{2}$ system shows $\mathrm{Ti}(\mathrm{IV})$ cation vacancies (vide infra) to balance the charge. Furthermore, laser irradiation creates some disordered (amorphous) zone and may further relax the structure. Both the effect of $\mathrm{Sb}$ doping and irradiation has a more complicated effect on the $A_{1 g}$ vibration. The $A_{1 g}$ peak in $\mathrm{TiO}_{2}$ is observed at $610 \mathrm{~cm}^{-1}$, but the presence of $\mathrm{Sb}$ increases the frequency to $614 \mathrm{~cm}^{-1}$, and the laser irradiation relaxes the structure shifting the peak position to $612 \mathrm{~cm}^{-1}$. Finally, the peak intensities decrease remarkably after laser irradiation, thus indicating that an amorphous layer of titanium oxide was formed on the surface of the irradiated sample.

UV-Vis Spectroscopy. The absorbance spectra-defined through the Kubelka-Munk function $F(R)$ as $(1-R)^{2} / 2 R$, where $R$ is the diffuse reflectance-of $\mathrm{TiO}_{2}, \mathrm{Sb}$-doped $\mathrm{TiO}_{2}$, and irradiated $\mathrm{Sb}$-doped $\mathrm{TiO}_{x}$ are shown in Figure 4 .

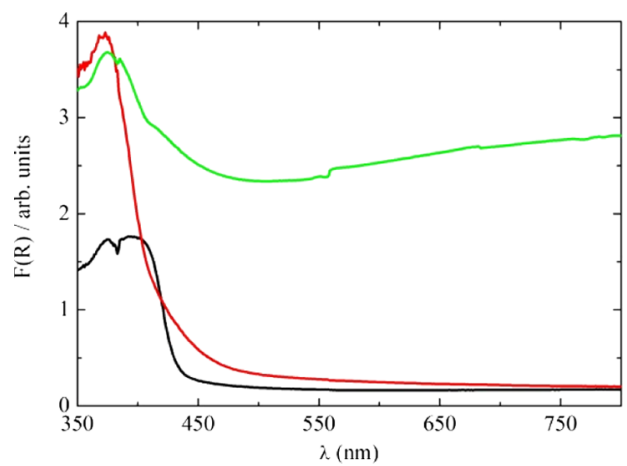

Figure 4. Absorbance spectra of $\mathrm{TiO}_{2}$ (black line), Sb-doped $\mathrm{TiO}_{2}$ (red line), and laser-irradiated Sb-doped $\mathrm{TiO}_{x}$ (green line).

The $\mathrm{TiO}_{2}$ sharp absorption at a wavelength lower than 450 $\mathrm{nm}$ is related to interband absorption at energies greater than that of the band gap. The effective band gap of about $2.8 \mathrm{eV}$ inferred from our measurements on nonirradiated, undoped $\mathrm{TiO}_{2}$ is somewhat lower than the value of $3.06 \mathrm{eV}$ usually quoted for the rutile polymorph of $\mathrm{TiO}_{2}$, possibly because of annealing at high temperature. This treatment is known to create oxygen vacancies and related $\mathrm{Ti}^{3+}$ defects. The presence of $\mathrm{Sb}$ in $\mathrm{TiO}_{2}$ introduces mid-gap states associated with segregated $\mathrm{Sb}$ at the surface and grain boundary interfaces, and absorption in the range $450-600 \mathrm{~nm}$ is enhanced. After laser irradiation in water, the sample turned gray, and there was a strong absorption across the complete visible range. This behavior indicates that new states due to the disorder and oxygen vacancies induced by the laser irradiation are formed in the band gap.
X-ray Photoelectron Spectroscopy. Figure 5 shows the normal emission core-level photoelectron spectra of the $1.5 \%$

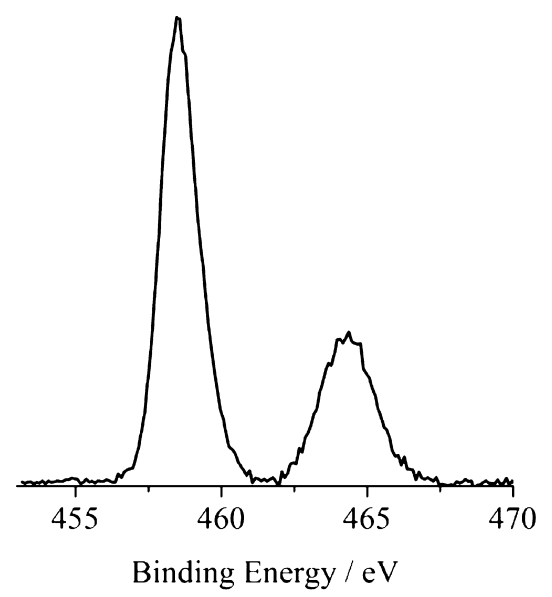

Figure 5. Monochromatized $\mathrm{Al} \mathrm{K} \alpha$-excited $\mathrm{X}$-ray photoelectron spectroscopy (XPS) of $1.5 \% \mathrm{Sb}$-doped $\mathrm{TiO}_{2}$ measured in the $\mathrm{Ti} 2 \mathrm{p}$ core-level binding energy region, at $45^{\circ}$ off-take angle relative to the surface plane.

Sb-doped $\mathrm{TiO}_{2}$ measured in the $\mathrm{Ti} 2 \mathrm{p}$ binding energy region. The $\mathrm{Ti} 2 \mathrm{p}_{3 / 2}$ and $2 \mathrm{p}_{1 / 2}$ states are at 458.5 and $464.4 \mathrm{eV}$, respectively.

It is important to keep in mind that XPS is a surface technique able to probe a thickness of about $30 \AA$ for $\mathrm{TiO}_{2}$. The core-level photoelectron spectra of $1.5 \% \mathrm{Sb}$-doped $\mathrm{TiO}_{2}$, measured in a binding energy region containing both $\mathrm{O} 1 \mathrm{~s}$ and $\mathrm{Sb} 3 \mathrm{~d}$ peaks, are shown in Figure 6.

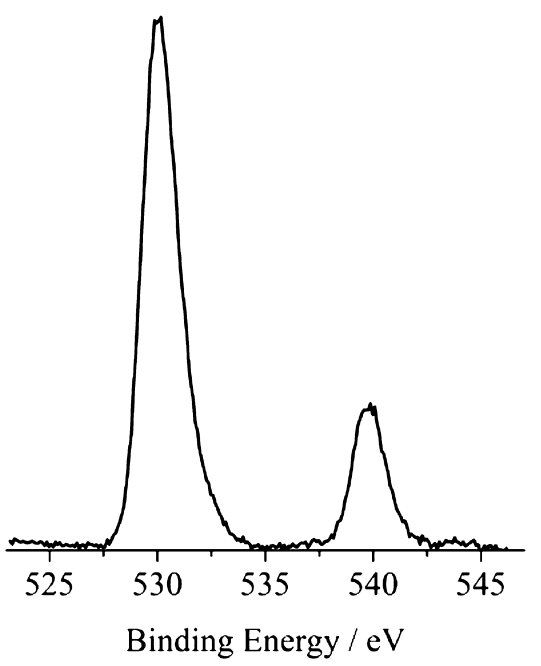

Figure 6. Monochromatized Al $\mathrm{K} \alpha$-excited XPS of $1.5 \% \mathrm{Sb}$-doped $\mathrm{TiO}_{2}$ measured in the $\mathrm{O} 1 \mathrm{~s}$ and $\mathrm{Sb} 3 \mathrm{~d}$ binding energy region, at a $45^{\circ}$ off-take angle relative to the surface plane.

It is well-known that $\mathrm{Sb}$ XPS binding energies cannot be used to diagnose the $\mathrm{Sb}$ oxidation state because any intrinsic shift between $\mathrm{Sb}(\mathrm{III})$ and $\mathrm{Sb}(\mathrm{V})$ is compensated by the site potential difference. ${ }^{57}$ Also, the Sn core-binding energies for $\mathrm{SnO}$ and $\mathrm{SnO}_{2}$ are identical. ${ }^{58}$ Because the $\mathrm{Sb} 3 \mathrm{~d}_{5 / 2}$ peak overlaps the $\mathrm{O} 1 \mathrm{~s}$ peak (at $530.0 \mathrm{eV}$ ) almost perfectly, only the intensity ratio between $S b 3 d_{3 / 2}$ and $\mathrm{Ti} 2 \mathrm{p}$ peaks was used to calculate the effective surface $\mathrm{Sb} / \mathrm{Ti}$ ratio. ${ }^{59,60}$ The obtained 
ratio $\mathrm{Sb} / \mathrm{Ti}$ is 0.16 , and this indicates a pronounced surface segregation of $\mathrm{Sb}^{41}$ This result is also in tune with the Sbinduced photoemission structure already found in the band gap at about $1.8 \mathrm{eV}$ by means of $\mathrm{He}-\mathrm{I} \mathrm{UV}$ photoemission spectroscopy (UPS, Figure S1). ${ }^{40,42}$ In fact, it emerged that, although $\mathrm{Sb}(\mathrm{V})$ ions substitute for bulk $\mathrm{Ti}(\mathrm{IV})$ ions, $\mathrm{Sb}(\mathrm{III})$ species, with a $(5 s-5 p)^{2}$ hybrid electron configuration and electron densities pointing out of the surface plane, are present at the surface. ${ }^{40,41}$ The XPS spectra of laser-irradiated Sbdoped $\mathrm{TiO}_{x}$ are almost identical to those of the nonirradiated material.

Photocatalysis. We further investigated the effect of $\mathrm{Sb}$ doping and laser irradiation on the photoactivity of the synthetized titanium oxides by following the discoloration of the methylene blue $(\mathrm{MB})$ dye. Data presented in Figure 7 show the absorbance at $665 \mathrm{~nm}$ of a $1.5 \times 10^{-5} \mathrm{M}$ water solution of $\mathrm{MB}$ as a function of $\mathrm{UV}$ irradiation time in the presence of the catalyst.

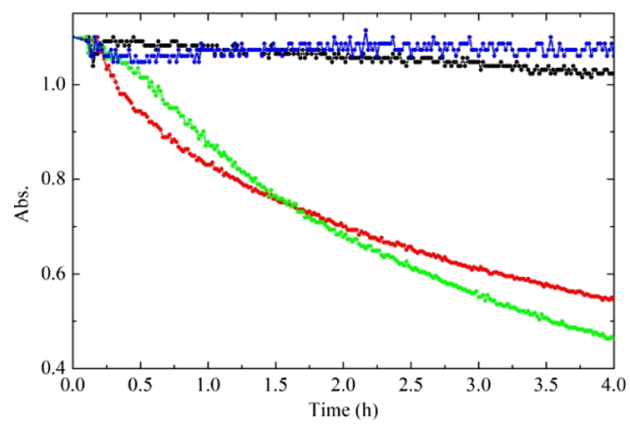

Figure 7. Absorbance at $665 \mathrm{~nm}$ of a $1.5 \times 10^{-5} \mathrm{M}$ water solution of $\mathrm{MB}$, as a function of UV (368 nm) irradiation time. Blue, black, red, and green curves are related to the $\mathrm{MB}$ reference solution (without catalyst), the $\mathrm{MB}$ solution in the presence of undoped $\mathrm{TiO}_{2}$, in the presence of $\mathrm{Sb}$-doped $\mathrm{TiO}_{2}$, and in the presence of $\mathrm{Sb}$-doped $\mathrm{TiO}_{x}$.

In the presence of the undoped $\mathrm{TiO}_{2}$, there is evidence of a very small $\mathrm{MB}$ absorbance decrease even after $4 \mathrm{~h}$. By contrast, both the Sb-doped $\mathrm{TiO}_{2}$ and the Sb-doped $\mathrm{TiO}_{x}$ act as effective photocatalysts for the discoloration of the MB. Moreover, the concentration of $\mathrm{MB}$ decreases following a firstorder kinetic. By fitting the curves with an exponential decay, we are able to extract the discoloration rate: the undoped $\mathrm{TiO}_{2}$ shows a discoloration rate constant of $0.015 \mathrm{~h}^{-1}$ (decay time $66 \mathrm{~h}$ ), whereas $\mathrm{Sb}$-doped $\mathrm{TiO}_{2}$ and $\mathrm{Sb}$-doped $\mathrm{TiO}_{x}$ measure $0.21 \mathrm{~h}^{-1}$ (decay time $4.7 \mathrm{~h}$ ) and $0.25 \mathrm{~h}^{-1}$ (decay time $4.0 \mathrm{~h}$ ), respectively. Therefore, the photoactivity increases of more than 1 order of magnitude (14-fold) using the Sb-doped $\mathrm{TiO}_{2}$ and a higher increase (16-fold) are observable after laser irradiation.

We also performed similar measurements to investigate the photocatalytic behavior when using visible light. To this purpose, we irradiated a $1.0 \times 10^{-5} \mathrm{M}$ water solution of $\mathrm{MB}$ (starting $\mathrm{Abs}=0.74$ ) in the presence of $1.5 \% \mathrm{Sb}$-doped $\mathrm{TiO}_{x}$ with a commercial OSRAM lamp (OSRAM 71, $8 \mathrm{~W}$ ) whose emission peak was centered at $453 \mathrm{~nm}$. In order to avoid the lamp UV component, a long-pass filter at $400 \mathrm{~nm}$ was used. Once more, we collected the absorbance at $665 \mathrm{~nm}$, and Figure 8 shows the different photocatalytic behavior when this solution was excited with UV $(368 \mathrm{~nm})$ or visible $(453 \mathrm{~nm})$ light. Clearly, the UV irradiation is more effective in promoting the photocatalysis, and, after $4 \mathrm{~h}$, the remaining $\mathrm{MB}$ was about

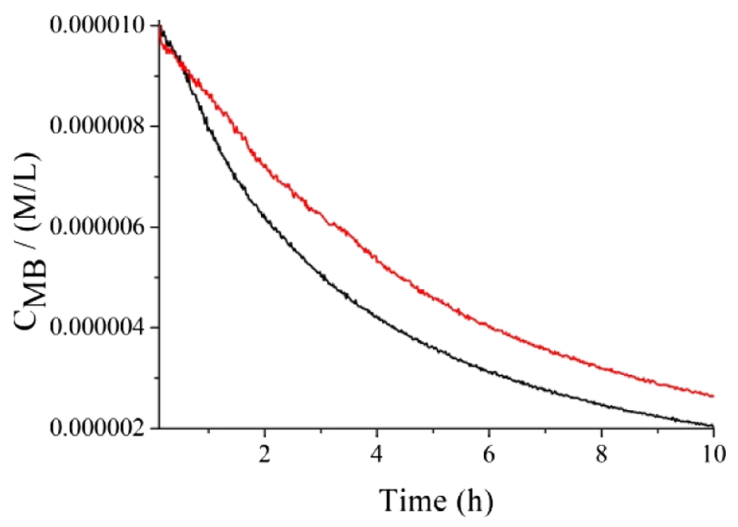

Figure 8. Absorbance at $665 \mathrm{~nm}$ of a $1.0 \times 10^{-5} \mathrm{M}$ water solution of $\mathrm{MB}(\mathrm{Abs}=0.74)$ in the presence of $1.5 \% \mathrm{Sb}$-doped $\mathrm{TiO}_{x}$, as a function of irradiation time: the black and red curves are related to the irradiation with UV and visible light, respectively. The experiments with a visible light source were performed using an OSRAM lamp (OSRAM 71, $8 \mathrm{~W}$ ) whose emission was centered at $453 \mathrm{~nm}$ with a full width at half-maximum (fwhm) lower than $50 \mathrm{~nm}$. The measured irradiance was approximately $1 \mathrm{~mW} / \mathrm{cm}^{2}$. A long-pass filter at $400 \mathrm{~nm}$ was used to avoid the UV illumination.

the $42 \%$ of the initial concentration, whereas, using visible light, the $53 \%$ of $\mathrm{MB}$ is still present. Even in the case of visible irradiation, the concentration of $\mathrm{MB}$ follows a first-order kinetic. The evaluated apparent decoloration rate constant is $0.16 \mathrm{~h}^{-1}$ (decay time $6.3 \mathrm{~h}$ ). After $10 \mathrm{~h}$, the difference in the $\mathrm{MB}$ residue is less pronounced, where the $20 \%$ of that is obtained using the UV and the $27 \%$ of that is observed using the visible irradiation. It is worth noting that under the $453 \mathrm{~nm}$ radiation, both $\mathrm{TiO}_{2}$ and $\mathrm{Sb}$-doped $\mathrm{TiO}_{2}$ show a negligible light absorption with a negligible visible photoactivity.

Interpretation of the photocatalytic activity: photoactivity is a complex phenomenon which involves several steps: (i) the generation of the electron-hole pairs, (ii) the separation and the diffusion of the charge carriers to the active sites on the surface, (iii) the interaction of holes with defects or with the MB lying on the surface-solution interphase, and (iv) the scavenging of the electrons. The observed photodegradation activity of $\mathrm{Sb}$-doped $\mathrm{TiO}_{2}$ must involve all these processes.

The photoactivity of bare $\mathrm{TiO}_{2}$ rutile, as argued by Fujishima, is correlated with the high oxidation power of the holes in the VB, in concomitance with an efficient mechanism for the scavenging of electrons mediated by the intercalation of hydrogen. ${ }^{61}$ The increase of the photoactivity of the Sb-doped $\mathrm{TiO}_{2}$ catalysts, in comparison with the undoped $\mathrm{TiO}_{2}$, is in tune with the increased light absorption observed in the UV spectrum (Figure 4). Nevertheless, this mechanism is not sufficient to explain the huge photoactivity increase (of 1 order of magnitude) experimentally observed.

By using the site stoichiometry and charge and mass conservation laws, we can write the following equations (using the Kroger-Vink notation) in order to balance the presence of $\mathrm{Sb}$ ion:

$$
\begin{aligned}
& \mathrm{Sb}_{2}{ }^{5+} \mathrm{O}_{5}{ }^{2-} \stackrel{2 \mathrm{TiO}_{2}}{\longrightarrow} 2 \mathrm{Sb}_{\mathrm{Ti}}{ }^{1+}+4 \mathrm{O}_{0}{ }^{x}+\frac{1}{2} \mathrm{O}_{2}+2 \mathrm{e}^{-} \\
& 2 \mathrm{Sb}_{2}{ }^{5+} \mathrm{O}_{5}{ }^{2-} \stackrel{5 \mathrm{TiO}_{2}}{\longrightarrow} 4 \mathrm{Sb}_{\mathrm{Ti}}{ }^{1+}+10 \mathrm{O}_{0}{ }^{x}+\mathrm{V}_{\mathrm{Ti}}{ }^{4-} \\
& \mathrm{Sb}_{2}{ }^{3+} \mathrm{O}_{3}{ }^{2-} \stackrel{2 \mathrm{TiO}_{2}}{\longrightarrow} 2 \mathrm{Sb}_{\mathrm{Ti}}{ }^{1-}+3 \mathrm{O}_{0}{ }^{x}+\mathrm{V}_{0}{ }^{++}
\end{aligned}
$$




$$
2 \mathrm{Sb}_{2}{ }^{3+} \mathrm{O}_{3}{ }^{2-} \stackrel{3 \mathrm{TiO}_{2}}{\longrightarrow} 3 \mathrm{Sb}_{\mathrm{Ti}}{ }^{1-}+6 \mathrm{O}_{0}{ }^{x}+\mathrm{Sb}_{\mathrm{I}}{ }^{3+}
$$

where $\mathrm{e}^{-}$in eq 1 represents the electrons in the $\mathrm{CB}, \mathrm{V}_{\mathrm{Ti}}$ in eq 2 indicates the Ti vacancies, $\mathrm{V}_{\mathrm{O}}$ in eq 3 accounts for the oxygen vacancies, and $\mathrm{Sb}_{\mathrm{I}}$ in eq 4 stands for the $\mathrm{Sb}$ interstitials. In this context, we already reported that $\mathrm{Sb}$ substitution in $\mathrm{TiO}_{2}$ involves a bulk substitution of $\mathrm{Sb}(\mathrm{V})$ ions onto the $\mathrm{Ti}(\mathrm{IV})$ sites and that the extra electron of the $\mathrm{Sb}(\mathrm{V})$ ion is largely compensated by the $\mathrm{Ti}(\mathrm{IV})$ vacancies, thus following eq $2 .^{40}$ Magnetic measurements show that in Sb-doped $\mathrm{TiO}_{2}$, only 3\% of the $\mathrm{Sb}$ dopant ions gives rise to unpaired spins. ${ }^{40}$ Therefore, the mode of compensation mainly included cation vacancies to give $\mathrm{Sb}(\mathrm{V})_{x} \mathrm{Ti}(\mathrm{IV})_{1-x(5 / 4)} \square_{x / 4} \mathrm{O}_{2}$ where $\square$ indicates a vacant $\mathrm{Ti}(\mathrm{IV})\left(\mathrm{V}_{\mathrm{Ti}}{ }^{4+}\right)$ site. In summary, if four $\mathrm{Sb}(\mathrm{V})$ ions $(20+$ net charge) substitute for four $\mathrm{Ti}(\mathrm{IV})$ ions (16+ net charge), a $\mathrm{Ti}(\mathrm{IV})$ vacancy (4+ net charge) maintains the electrical neutrality in the system following eq $2 .{ }^{40}$

$\mathrm{Sb}$ (III) species, having a formal $5 \mathrm{~s}^{2}$ electron configuration, are accommodated at the surface, where the empty $5 p$ states hybridize with the spherically symmetric 5 s states. The hybrids sit above the main O $2 p$ VB edge and are therefore capable of trapping photogenerated holes at sites on the catalyst surface, where the holes are optimally placed to oxidize surfaceadsorbed organic species. The net effect is an increase of the activity of 14-fold.

The laser-irradiated $\mathrm{Sb}$-doped $\mathrm{TiO}_{x}$ sample shows an even higher photoactivity than the nonirradiated $\mathrm{Sb}$-doped $\mathrm{TiO}_{2}$. Laser irradiation induces a profound modification of the material: it realizes a disordered (amorphous) layer on the surface of the $\mathrm{Sb}$-doped $\mathrm{TiO}_{2}{ }^{51}$ During the laser irradiation in water, a high-temperature and high-pressure plasma is produced. Moreover, heat diffusion during the first $10 \mathrm{~ns}$ of irradiation realizes a bubble (called cavitation bubble) that limits the plasma expansion with a consequent lingering of high temperature (for several microseconds). ${ }^{62}$ The cavitation bubble has a complex temporal evolution. The shrinking and the collapse of the bubble realize a new high-pressure and hightemperature state. ${ }^{63}$ These conditions make possible the synthesis of a partially amorphous, hydrogenated, reduced material. Hydrogen acts as a donor when intercalated the $\mathrm{TiO}_{2}$ structure and might be expected to produce the occupied states near the conduction band minimum (CBM). Following the same argument proposed by Fujishima in 2008, $\mathrm{H}$ induces a local surface band bending, favors the scavenging of the electrons during the photocatalytical process, and thus enhances the activity. ${ }^{61}$ However, in high concentrations, it may cause a distortion of the oxygen environment, thus creating intragap states close to the $\mathrm{VB}^{61}$ In this case, the states near the CBM are weakly influenced upon the $\mathrm{O}$ bound distortion being based on the Ti $3 \mathrm{~d}$ states. ${ }^{46,64}$ This distortion may create surface trap states enhancing the activity.

Previous studies on the photocatalytic activity of $\mathrm{Sb}-\mathrm{TiO}_{2}$ have been already reported with no separation of the UV and visible contributions, and some microstructural variations of the catalysts were involved in the interpretation of the photocatalytic activity upon the variation of the $\mathrm{Sb}$ doping level. ${ }^{35}$ By contrast, in our previous studies, using UPS, lowenergy electron diffraction, XPS, TEM, and superconducting quantum interference device magnetometry, we demonstrated that at low Sb doping levels ( $\sim 1$ at. \%), a well-defined peak appears at $1.8 \mathrm{eV}$ in the band gap due to the lone pairs of the segregated of $\mathrm{Sb}(\mathrm{III})$ surface states. ${ }^{40-42}$ Moreover, at these low doping levels, $\mathrm{Sb}$ strongly segregates only to the topmost ionic layer, whereas at higher Sb doping levels (3-10 at. \%), the $\mathrm{Sb}$ segregation involves more ionic layers having a lower $\mathrm{Sb}$ content per layer and the intensity of the UPS $\mathrm{Sb}$ (III) surface states significantly reduced. Therefore, we have chosen to study the photocatalysis of $1.5 \% \mathrm{Sb}$-doped $\mathrm{TiO}_{2}$ because it possesses the highest surface concentration of these $\mathrm{Sb}$ lone pair states that cause a visible region absorption and allow the formation of surface site traps for photogenerated holes.

Recent studies have shown that the improvement of the photocatalytic properties of the $\mathrm{TiO}_{2}$ doped with transition metals can also be seen in terms of modification of density of state of the VB that includes the change in the d-orbital shape and broadening. ${ }^{65}$

Our present study does not involve transition metals as dopants, but surface $\mathrm{Sb}$ gives rise to lone pair states just above the VB maximum. This both promotes the visible region absorption and gives rise to sites on the surface which can trap photogenerated holes. Moreover, it is argued that the intercalation of hydrogen due to laser ablation can produce states close to the VB edge and able to scavenge electrons. ${ }^{61}$

\section{CONCLUSIONS}

In the present study, we synthetized and characterized some $1.5 \% \mathrm{Sb}$-doped $\mathrm{TiO}_{2}$ polycrystalline materials. Antimony doping in $\mathrm{TiO}_{2}$ produces new occupied states above the VB maximum, thus reducing the effective band gap and increasing the absorption in the visible range. A further increase of the absorption was observed after laser irradiation in water because of the realization of a continuum of states in the band gap.

Moreover, doping of $\mathrm{TiO}_{2}$ with $\mathrm{Sb}$ ions resulted in an increased $\mathrm{MB}$ degradation photoactivity of more than 1 order of magnitude with respect to the undoped sample. This enhancement was discussed in terms of increased light absorption and trapping of holes at the $\mathrm{Sb}$ surface states. A further increase of the activity observed in the laser-irradiated sample was interpreted in terms of the formation of deep states that further favors the trapping of holes near the surface of the Sb-doped $\mathrm{TiO}_{x}$ as well as the scavenging of electrons by intercalated hydrogen. We are confident that the $\mathrm{Sb}-\mathrm{TiO}_{2}$ may be used as an efficient and sensitive photocatalyst for water purification. ${ }^{66-71}$

\section{EXPERIMENTAL DETAILS}

Materials and Methods. The undoped and $1.5 \% \mathrm{Sb}$ doped $\mathrm{TiO}_{2}$ samples were prepared as already described. ${ }^{40}$ The resulting powder was pelletized between $\mathrm{WC}$ dies and annealed for 5 days at $1000{ }^{\circ} \mathrm{C}$ in an alumina crucible in order to confer to $\mathrm{Sb}$ ions enough mobility to substitute for $\mathrm{T}(\mathrm{IV})$ in lattice positions. Annealing at temperatures up to 800 ${ }^{\circ} \mathrm{C}$ resulted in $\mathrm{TiO}_{2}$ and $\mathrm{Sb}_{2} \mathrm{O}_{4}$ mixed phases. A similar procedure without the addition of the $\mathrm{Sb}$ solution was followed to obtain undoped $\mathrm{TiO}_{2}$ pellets.

The preparation of amorphous Sb-doped $\mathrm{TiO}_{x}$ was achieved by irradiating an Sb-doped $\mathrm{TiO}_{2}$ pellet immersed in deionized Milli-Q water (resistivity $18 \mathrm{M} \Omega \cdot \mathrm{cm}$ ) within a quartz cuvette. The radiation source was a Nd:YAG pulsed laser (Giant G79030 ) at $532 \mathrm{~nm}, 10 \mathrm{~Hz}$ repetition rate, and $80 \mathrm{~mJ}$ energy per pulse. We used the Van Cittert-Zernike theorem results to have a smooth laser spatial profile: the path length of the laser and the diameter of the laser beam have been adjusted to $30 \mathrm{~m}$ and $4 \mathrm{~mm}$, respectively. The obtained diffraction pattern has a coherence area of about $4 \mathrm{~mm}$ in diameter by smoothing of the 
laser beam hot spots. A Gaussian intensity profile with a fwhm of $1 \mathrm{~mm}$ was used in the present experiment. ${ }^{21}$ Samples were irradiated at a fluence of $2 \mathrm{~J} / \mathrm{cm}^{2}$ for several minutes.

Characterization of Sb-Doped $\mathrm{TiO}_{2}$. The RBS measurements were carried out using a $2 \mathrm{MeV} \mathrm{He}^{+}$beam with a scattering angle of $165^{\circ}$ in a normal incidence. The RUMP software was employed for the analysis of the RBS data.

The phase structure of the samples was determined by the grazing angle $\left(0.5^{\circ}\right) \mathrm{XRD}$ using a Bruker D-9000 instrument incorporating a $\mathrm{Cu} \mathrm{K} \alpha$ anode and a Bruker suite software for the analysis of the diffraction patterns.

The Raman measurements were performed in a backscattering configuration using a $\mathrm{HeNe}$ laser, with the analysis of the scattered radiation by a $32 \mathrm{~cm}$ focal length monochromator incorporating 1800 grooves $/ \mathrm{mm}$ diffraction grating. The penetration length of the laser into $\mathrm{TiO}_{2}$ is estimated to be some hundreds of nanometers because of the multiple scattering on the surface.

The UV/visible reflectance spectra were collected using a PerkinElmer LAMBDA 40 spectrometer in the 350-900 nm with an integrating sphere (Labsphere 20). The absorbance was calculated with the formula: $A=100-R$, where $R$ is the integrated reflectivity in percentage.

The XPS measurements were performed with a PHI 5000 VersaProbe II (the base pressure of the main chamber is $1 \times$ $10^{-8} \mathrm{~Pa}$ ) using monochromatized $\mathrm{Al} \mathrm{K} \alpha$ radiation. Calibration and background removal were performed as already described. ${ }^{59,72}$

Photocatalytic Activity. In order to evaluate the photocatalytic activity, UV photodegradation tests were carried out, based on the discoloration of the MB dye. In particular, $20 \mu \mathrm{L}$ of a $\mathrm{MB}$ water solution ( $0.05 \mathrm{wt} \%$ ) was added to $2 \mathrm{~mL}$ of water to give a final concentration of $1.5 \times 10^{-5} \mathrm{M}$ and an absorbance of $1.1\left(\varepsilon=7.4 \times 10^{4} \mathrm{M}^{-1} \mathrm{~cm}^{-1}\right)$. The pellet was vertically placed into this resulting solution that was not stirred, to avoid any possible surface pellet modification because of the bumps between the magnet and the pellet. A standard $1 \mathrm{~cm}^{2}$ cuvette was used for the transmittance measurements.

The transmittance of the $\mathrm{MB}$ solution at the adsorption peak of the dye $(665 \mathrm{~nm})$ was measured as a function of time, during the UV irradiation, using a homemade apparatus composed of a $665 \mathrm{~nm} \mathrm{LED,} \mathrm{red} \mathrm{sensitive} \mathrm{photodiode}$ (Hamamatsu S6430-01 with a spectral response between 600 and $700 \mathrm{~nm}$ ), and dedicated electronics. The absorption of the solution as a function of time was calculated by using the logarithm of the transmittance. The output of the UV light source (TL $8 \mathrm{~W}$ BLB 1FM, Philips) was centered at $368 \mathrm{~nm}$ with a fwhm lower than $10 \mathrm{~nm}$ and the measured irradiance was about $1.1 \mathrm{~mW} / \mathrm{cm}^{2}$.

In addition, similar experiments have been performed with a visible light source using an OSRAM lamp (OSRAM 71, $8 \mathrm{~W}$ ) whose emission was centered at $453 \mathrm{~nm}$ with a fwhm lower than $50 \mathrm{~nm}$. The measured irradiance was approximately 1 $\mathrm{mW} / \mathrm{cm}^{2}$. A long-pass filter at $400 \mathrm{~nm}$ was used to avoid UV illumination.

The catalytic measurements have been performed four times with no variation in the pellet performance. At the end of each cycle, it was enough to irradiate the $\mathrm{Sb}-\mathrm{TiO}_{2}$ with a UV lamp for cleaning and to restore the initial photocatalytic activity.

Finally, we want to stress that $\mathrm{Sb}(\mathrm{V})$ substitutes for $\mathrm{T}(\mathrm{IV})$ in lattice positions, and the obtained $\mathrm{Sb}$-doped system does not release any significant $\mathrm{Sb}$ ion in water $(<3 \mu \mathrm{g} / \mathrm{L})$, as measured by inductively coupled plasma emission spectroscopy, whereas pure $\mathrm{Sb}_{2} \mathrm{O}_{3}$ has a solubility of $\sim 370 \mu \mathrm{g} / \mathrm{L}$, at room temperature. This fact indicates that the $\mathrm{Sb}-\mathrm{TiO}_{2}$ photocatalyst is not toxic.

\section{ASSOCIATED CONTENT}

\section{S Supporting Information}

The Supporting Information is available free of charge on the ACS Publications website at DOI: 10.1021/acsomega.8b01452.

UPS $\mathrm{He}-\mathrm{I}$ spectrum of $1.5 \% \mathrm{Sb}-\mathrm{TiO}_{2}(\mathrm{PDF})$

\section{AUTHOR INFORMATION}

\section{Corresponding Authors}

*E-mail: russell.egdell@chem.ox.ac.uk. Phone: +44(0)1865275965. Fax: +44(0) 1865-272690 (R.G.E.).

*E-mail: mariagrazia.grimaldi@ct.infn.it. Phone: +39-0953785352. Fax: +39-095-337938 (M.G.G.).

*E-mail: agulino@unict.it. Phone: +39-095-7385067. Fax: +39-095-580138 (A.G.).

\section{ORCID $\odot$}

Antonino Gulino: 0000-0002-6850-3080

\section{Author Contributions}

This manuscript was written through contributions from all authors. All authors have given approval to the final version of the manuscript.

\section{Notes}

The authors declare no competing financial interest.

\section{ACKNOWLEDGMENTS}

The authors thank the "Piano della Ricerca 2018" of the University of Catania for financial support.

\section{REFERENCES}

(1) Asahi, R.; Morikawa, T.; Ohwaki, T.; Aoki, K.; Taga, Y. Visiblelight photocatalysis in nitrogen-doped titanium oxides. Science 2001, 293, 269-271.

(2) Di Valentin, C.; Finazzi, E.; Pacchioni, G.; Selloni, A.; Livraghi, S.; Paganini, M. C.; Giamello, E. N-doped TiO2: Theory and experiment. Chem. Phys. 2007, 339, 44-56.

(3) Qiu, X.; Burda, C. Chemically synthesized nitrogen-doped metal oxide nanoparticles. Chem. Phys. 2007, 339, 1-10.

(4) Linsebigler, A. L.; Lu, G.; Yates, J. T. Photocatalysis on TiO2 Surfaces: Principles, Mechanisms, and Selected Results. Chem. Rev. 1995, 95, 735-758.

(5) Wong, J. C. S.; Linsebigler, A.; Lu, G.; Fan, J.; Yates, J. T. J. Photooxidation of $\mathrm{CH} 3 \mathrm{Cl}$ on $\mathrm{TiO} 2(110)$ Single Crystal and Powdered TiO2 Surfaces. J. Phys. Chem. 1995, 99, 335-344.

(6) Carp, O.; Huisman, C. L.; Reller, A. Photoinduced reactivity of titanium dioxide. Prog. Solid State Chem. 2004, 32, 33-177.

(7) Oropeza, F. E.; Davies, B.; Palgrave, R. G.; Egdell, R. G. Electronic basis of visible region activity in high area $\mathrm{Sn}$-doped rutile TiO2 photocatalysts. Phys. Chem. Chem. Phys. 2011, 13, 7882-7891.

(8) Oropeza, F. E.; Zhang, K. H. L.; Palgrave, R. G.; Regoutz, A.; Egdell, R. G.; Allen, J. P.; Galea, N. M.; Watson, G. W. Electronic Structure of Epitaxial Sn-Doped Anatase Grown on SrTiO3(001) by Dip Coating. J. Phys. Chem. C 2013, 117, 15221-15228.

(9) Clendenen, R. L.; Drickamer, H. G. Lattice Parameters of Nine Oxides and Sulfides as a Function of Pressure. J. Chem. Phys. 1966, 44, $4223-4228$

(10) Rao, K. V. K.; Naidu, S. V. N.; Iyengar, L. Thermal expansion of rutile and anatase. J. Am. Ceram. Soc. 1970, 53, 124-126. 
(11) Madelung, O. Semiconductors: Physics of Non-Tetrahedrally Bonded Binary Compounds II. Landon-Bornstein New Science, Group III; Springer Verlag: Berlin, 1984; Part f, Vol. 17.

(12) Tang, H.; Lévy, F.; Berger, H.; Schmid, P. E. Urbach tail of anataseTiO2. Phys. Rev. B: Condens. Matter Mater. Phys. 1995, 52, 7771-7774.

(13) Mo, S.-D.; Ching, W. Y. Electronic and optical properties of three phases of titanium dioxide: Rutile, anatase, and brookite. Phys. Rev. B: Condens. Matter Mater. Phys. 1995, 51, 13023-13032.

(14) Labat, F.; Baranek, P.; Domain, C.; Minot, C.; Adamo, C. Density functional theory analysis of the structural and electronic properties of $\mathrm{TiO} 2$ rutile and anatase polytypes: Performances of different exchange-correlation functionals. J. Chem. Phys. 2007, 126, 154703.

(15) Scanlon, D. O.; Dunnill, C. W.; Buckeridge, J.; Shevlin, S. A.; Logsdail, A. J.; Woodley, S. M.; Catlow, C. R. A.; Powell, M. J.; Palgrave, R. G.; Parkin, I. P.; Watson, G. W.; Keal, T. W.; Sherwood, P.; Walsh, A.; Sokol, A. A. Band alignment of rutile and anatase $\mathrm{TiO} 2$. Nat. Mater. 2013, 12, 798-801.

(16) Di Valentin, C.; Pacchioni, G.; Selloni, A.; Livraghi, S.; Giamello, E. Characterization of Paramagnetic Species in N-Doped TiO2Powders by EPR Spectroscopy and DFT Calculations. J. Phys. Chem. B 2005, 109, 11414-11419.

(17) Livraghi, S.; Paganini, M. C.; Giamello, E.; Selloni, A.; Di Valentin, C.; Pacchioni, G. Origin of photoactivity of nitrogen-doped titanium dioxide under visible light. J. Am. Chem. Soc. 2006, 128, $15666-15671$.

(18) Napoli, F.; Chiesa, M.; Livraghi, S.; Giamello, E.; Agnoli, S.; Granozzi, G.; Pacchioni, G.; Di Valentin, C. The nitrogen photoactive centre in $\mathrm{N}$-doped titanium dioxide formed via interaction of $\mathrm{N}$ atoms with the solid. Nature and energy level of the species. Chem. Phys. Lett. 2009, 477, 135-138.

(19) Livraghi, S.; Votta, A.; Paganini, M. C.; Giamello, E. The nature of paramagnetic species in nitrogen doped $\mathrm{TiO} 2$ active in visible light photocatalysis. Chem. Commun. 2005, 0, 498-500.

(20) Livraghi, S.; Chierotti, M. R.; Giamello, E.; Magnacca, G.; Paganini, M. C.; Cappelletti, G.; Bianchi, C. L. Nitrogen-Doped Titanium Dioxide Active in Photocatalytic Reactions with Visible Light: A Multi-Technique Characterization of Differently Prepared Materials. J. Phys. Chem. C 2008, 112, 17244-17252.

(21) Zhao, Y.; Qiu, X.; Burda, C. The Effects of Sintering on the Photocatalytic Activity of N-Doped TiO2Nanoparticles. Chem. Mater. 2008, 20, 2629-2636.

(22) Irie, H.; Watanabe, Y.; Hashimoto, K. Nitrogen-Concentration Dependence on Photocatalytic Activity of TiO2-xNxPowders. J. Phys. Chem. B 2003, 107, 5483-5486.

(23) In, S.; Orlov, A.; Berg, R.; García, F.; Pedrosa-Jimenez, S.; Tikhov, M. S.; Wright, D. S.; Lambert, R. M. Effective Visible LightActivated B-Doped and B,N-Codoped TiO2Photocatalysts. J. Am. Chem. Soc. 2007, 129, 13790-13791.

(24) Geng, H.; Yin, S.; Yang, X.; Shuai, Z.; Liu, B. Geometric and electronic structures of the boron-doped photocatalyst TiO2. J. Phys.: Condens. Matter 2006, 18, 87-96.

(25) Irie, H.; Watanabe, Y.; Hashimoto, K. Carbon-doped Anatase TiO2Powders as a Visible-light Sensitive Photocatalyst. Chem. Lett. 2003, 32, 772-773.

(26) Halford, B. FLUID BOUNDARIES. Chem. Eng. News 2003, 81, 13.

(27) Yu, J. C.; Ho, W.; Yu, J.; Yip, H.; Wong, P. K.; Zhao, J. Efficient Visible-Light-Induced Photocatalytic Disinfection on Sulfur-Doped Nanocrystalline Titania. Environ. Sci. Technol. 2005, 39, 1175-1179.

(28) Umebayashi, T.; Yamaki, T.; Tanaka, S.; Asai, K. Visible LightInduced Degradation of Methylene Blue on S-doped TiO2. Chem. Lett. 2003, 32, 330-331.

(29) Klosek, S.; Raftery, D. Visible Light Driven V-Doped TiO2Photocatalyst and Its Photooxidation of Ethanol. J. Phys. Chem. B 2001, 105, 2815-2819.

(30) Niishiro, R.; Kato, H.; Kudo, A. Nickel and either tantalum or niobium-codoped $\mathrm{TiO} 2$ and $\mathrm{SrTiO} 3$ photocatalysts with visible-light response for $\mathrm{H} 2$ or $\mathrm{O} 2$ evolution from aqueous solutions. Phys. Chem. Chem. Phys. 2005, 7, 2241-2245.

(31) Dholam, R.; Patel, N.; Adami, M.; Miotello, A. Hydrogen production by photocatalytic water-splitting using $\mathrm{Cr}$ - or $\mathrm{Fe}$-doped $\mathrm{TiO} 2$ composite thin films photocatalyst. Int. J. Hydrogen Energy 2009, 34, 5337-5346.

(32) Oropeza, F. E.; Egdell, R. G. Control of valence states in Rhdoped $\mathrm{TiO} 2$ by $\mathrm{Sb}$ co-doping: A study by high resolution $\mathrm{X}$-ray photoemission spectroscopy. Chem. Phys. Lett. 2011, 515, 249-253.

(33) Nolan, M. First-Principles Prediction of New Photocatalyst Materials with Visible-Light Absorption and Improved Charge Separation: Surface Modification of Rutile $\mathrm{TiO} 2$ with Nanoclusters of $\mathrm{MgO}$ and $\mathrm{Ga2O}$. ACS Appl. Mater. Interfaces 2012, 4, 5863-5871.

(34) Kumar, A. S.; Nagaraja, K. K.; Huang, N. M.; Nagaraja, H. S. Preparation, characterization and photoelectrochemical properties of hydrophilic Sn doped TiO2 nanostructures. Mater. Lett. 2014, 123, 149-152.

(35) Moon, J.; Takagi, H.; Fujishiro, Y.; Awano, M. Preparation and characterization of the Sb-doped $\mathrm{TiO}_{2}$ photocatalysts. J. Mater. Sci. 2001, 36, 949-955.

(36) Cox, P. A.; Egdell, R. G.; Harding, C.; Orchard, A. F.; Patterson, W. R.; Tavener, P. J. Free-electron behaviour of carriers in antimony-doped tin(IV) oxide: A study by electron spectroscopy. Solid State Commun. 1982, 44, 837-839.

(37) Cox, P. A.; Egdell, R. G.; Harding, C.; Patterson, W. R.; Tavener, P. J. Surface properties of antimony doped tin(IV) oxide: A study by electron spectroscopy. Surf. Sci. 1982, 123, 179-203.

(38) Egdell, R. G.; Flavell, W. R.; Tavener, P. Antimony-doped tin(IV) oxide: Surface composition and electronic structure. J. Solid State Chem. 1984, 51, 345-354.

(39) Bhachu, D. S.; Egdell, R. G.; Sankar, G.; Carmalt, C. J.; Parkin, I. P. Electronic properties of antimony-doped anatase $\mathrm{TiO} 2$ thin films prepared by aerosol assisted chemical vapour deposition. J. Mater. Chem. C 2017, 5, 9694-9701.

(40) Gulino, A.; Taverner, A. E.; Warren, S.; Harris, P.; Egdell, R. G. A photoemission study of Sb-doped TiO2. Surf. Sci. 1994, 315, 351361.

(41) Gulino, A.; Condorelli, G. G.; Fragalà, I.; Egdell, R. G. Surface segregation of $\mathrm{Sb}$ in doped $\mathrm{TiO} 2$ rutile. Appl. Surf. Sci. 1995, 90, 289295.

(42) Taverner, A. E.; Gulino, A.; Egdell, R. G.; Tate, T. J. A photoemission study of electron states in Sb-ion implanted TiO2(110). Appl. Surf. Sci. 1995, 90, 383-387.

(43) He, L.; Mao, C.; Brasino, M. D.; Harguindey, A.; Park, W.; Goodwin, A. P.; Cha, J. N. $\mathrm{TiO}_{2}$ Capped Gold Nanorods for Plasmon-Enhanced Production of Reactive Oxygen Species and Photothermal Delivery of Chemotherapeutic Agents. ACS Appl. Mater. Interfaces 2018, 10, 27965.

(44) Saeed, M.; Iqbal, M. Z.; Ren, W.; Xia, Y.; Liu, C.; Khan, W. S.; $\mathrm{Wu}, \mathrm{A}$. Controllable synthesis of $\mathrm{Fe} 3 \mathrm{O} 4$ nanoflowers: enhanced imaging guided cancer therapy and comparison of photothermal efficiency with black-TiO2. J. Mater. Chem. B 2018, 6, 3800-3810.

(45) Wang, J.; Qian, S.; Liu, X.; Xu, L.; Miao, X.; Xu, Z.; Cao, L.; Wang, H.; Jiang, X. M2 macrophages contribute to osteogenesis and angiogenesis on nanotubular $\mathrm{TiO} 2$ surfaces. J. Mater. Chem. B 2017, 5, 3364-3376.

(46) Chen, X.; Liu, L.; Yu, P. Y.; Mao, S. S. Increasing solar absorption for photocatalysis with black hydrogenated titanium dioxide nanocrystals. Science 2011, 331, 746-750.

(47) Liu, Y.; Zhang, Y.; Wang, L.; Yang, G.; Shen, F.; Deng, S.; Zhang, X.; He, Y.; Hu, Y.; Chen, X. Fast and Large-Scale Anodizing Synthesis of Pine-Cone TiO2 for Solar-Driven Photocatalysis. Catalysts 2017, 7, 229.

(48) Zimbone, M.; Cacciato, G.; Sanz, R.; Carles, R.; Gulino, A.; Privitera, V.; Grimaldi, M. G. Black $\mathrm{TiO} x$ photocatalyst obtained by laser irradiation in water. Catal. Commun. 2016, 84, 11-15.

(49) Zimbone, M.; Cacciato, G.; Buccheri, M. A.; Sanz, R.; Piluso, N.; Reitano, R.; La Via, F.; Grimaldi, M. G.; Privitera, V. Photocatalytical activity of amorphous hydrogenated $\mathrm{TiO} 2$ obtained 
by pulsed laser ablation in liquid. Mater. Sci. Semicond. Process. 2016, $42,28-31$.

(50) Spucches, D.; Zimbone, M.; Cacciato, G.; Ruffino, F.; Privitera, V.; Grimaldi, M. G. Optical and morphological evolution of black $\mathrm{TiO}_{\mathrm{x}}$ synthesized in water by Nd:YAG laser. Phys. Status Solidi C 2017, 14, 1700134.

(51) Zimbone, M.; Cacciato, G.; Boutinguiza, M.; Gulino, A.; Cantarella, M.; Privitera, V.; Grimaldi, M. G. Hydrogenated black$\mathrm{TiO}_{\mathrm{x}}$ : A facile and scalable synthesis for environmental water purification. Catal. Today 2018, DOI: 10.1016/j.cattod.2018.03.040.

(52) ASTM File 21-1272. Database PDF (Powder Diffraction File).

(53) Zhang, H.; Banfield, J. F. Thermodynamic analysis of phase stability of nanocrystalline titania. J. Mater. Chem. 1998, 8, 20732076.

(54) Hanaor, D. A. H.; Sorrell, C. C. Review of the anatase to rutile phase transformation. J. Mater. Sci. 2011, 46, 855-874.

(55) Lukačević, I.; Gupta, S. K.; Jha, P. K.; Kirin, D. Lattice dynamics and Raman spectrum of rutile $\mathrm{TiO}_{2}$ : the role of soft phonon modes in pressure induced phase transition. Mater. Chem. Phys. 2012, 137, 282-289.

(56) Cacciato, G.; Bayle, M.; Pugliara, A.; Bonafos, C.; Zimbone, M.; Privitera, V.; Grimaldi, M. G.; Carles, R. Enhancing carrier generation in $\mathrm{TiO} 2$ by a synergistic effect between plasmon resonance in $\mathrm{Ag}$ nanoparticles and optical interference. Nanoscale 2015, 7, 1346813476.

(57) Orchard, A. F.; Thornton, G. Evidence of 'mixed-valency' character in the $\mathrm{X}$-ray photoelectron spectra of $\alpha$-diantimony tetraoxide and barium bismuth trioxide. J. Chem. Soc., Dalton Trans. 1977, $1238-1240$.

(58) Lau, C. L.; Wertheim, G. K. Oxidation of tin: An ESCA study. J. Vac. Sci. Technol. 1978, 15, 622-624.

(59) Briggs, D.; Grant, J. T. Surface Analysis by Auger and X-ray Photoelectron Spectroscopy; IMP: Chichester, U.K., 2003.

(60) Seah, M. P. The quantitative analysis of surfaces by XPS: A review. Surf. Interface Anal. 1980, 2, 222-239.

(61) Fujishima, A.; Zhang, X.; Tryk, D. TiO2 photocatalysis and related surface phenomena. Surf. Sci. Rep. 2008, 63, 515-582.

(62) Sasaki, K. Dynamics of liquid-phase laser ablation. In Laser Ablation in Liquids: Principles and Applications in the Preparation of Nanomaterials; Yang, G., Ed.; CRC Press, 2009; pp 269-295.

(63) Zhang, D.; Gökce, B.; Barcikowski, S. Laser synthesis and processing of colloids: fundamentals and applications. Chem. Rev. 2017, 117, 3990-4103.

(64) Chen, X.; Liu, L.; Huang, F. Black titanium dioxide (TiO2) nanomaterials. Chem. Soc. Rev. 2015, 44, 1861-1885.

(65) Md Saad, S. K.; Umar, A. A.; Umar, M. I. A.; Tomitori, M.; Rahman, M. Y. A.; Salleh, M. M.; Oyama, M. Two-Dimensional, Hierarchical Ag-Doped $\mathrm{TiO}_{2}$ Nanocatalysts: Effect of the Metal Oxidation State on the Photocatalytic Properties. ACS Omega 2018, 3, 2579-2587.

(66) Hu, S.; Shaner, M. R.; Beardslee, J. A.; Lichterman, M.; Brunschwig, B. S.; Lewis, N. S. Amorphous $\mathrm{TiO} 2$ coatings stabilize $\mathrm{Si}$, $\mathrm{GaAs}$, and $\mathrm{GaP}$ photoanodes for efficient water oxidation. Science 2014, 344, 1005-1009.

(67) Liu, M.; Qiu, X.; Miyauchi, M.; Hashimoto, K. Cu(II) Oxide Amorphous Nanoclusters Grafted Ti3+Self-Doped TiO2: An Efficient Visible Light Photocatalyst. Chem. Mater. 2011, 23, 5282-5286.

(68) Yu, H.; Irie, H.; Hashimoto, K. Conduction Band Energy Level Control Of Titanium Dioxide: Toward An Efficient Visible-LightSensitive Photocatalyst. J. Am. Chem. Soc. 2010, 132, 6898.

(69) Shannon, M. A.; Bohn, P. W.; Elimelech, M.; Georgiadis, J. G.; Mariñas, B. J.; Mayes, A. M. Science and technology for water purification in the coming decades. Nature 2008, 452, 301-310.

(70) Hashimoto, K.; Irie, H.; Fujishima, A. TiO2Photocatalysis: A Historical Overview and Future Prospects. Jpn. J. Appl. Phys. 2005, 44, $8269-8285$.

(71) Fujishima, A.; Honda, K. Electrochemical Photolysis of Water at a Semiconductor Electrode. Nature 1972, 238, 37-38.
(72) Gulino, A. Structural and Electronic Characterization of SelfAssembled Molecular Nanoarchitectures by X-ray Photoelectron Spectroscopy. Anal. Bioanal. Chem. 2013, 405, 1479-1495. 